\title{
Theory of Magnetic Ordering in the Heavy Rare Earths: $A b$ Initio Electronic Origin of Pair- and Four-Spin Interactions
}

\author{
Eduardo Mendive-Tapia* and Julie B. Staunton \\ Department of Physics, University of Warwick, Coventry CV4 7AL, United Kingdom \\ (Received 21 October 2016; published 11 May 2017)
}

\begin{abstract}
We describe a disordered local moment theory for long-period magnetic phases and investigate the temperature and magnetic field dependence of the magnetic states in the heavy rare earth elements (HREs), namely, paramagnetic, conical and helical antiferromagnetic (HAFM), fan, and ferromagnetic (FM) states. We obtain a generic HRE magnetic phase diagram which is consequent on the response of the common HRE valence electronic structure to $f$-electron magnetic moment ordering. The theory directly links the first-order HAFM-FM transition to the loss of Fermi surface nesting, induced by this magnetic ordering, as well as provides a template for analyzing the other phases and exposing where $f$-electron correlation effects are particularly intricate. Gadolinium, for a range of hexagonal, close-packed lattice constants $c$ and $a$, is the prototype, described $a b$ initio, and applications to other HREs are made straightforwardly by scaling the effective pair and quartic local moment interactions that emerge naturally from the theory with de Gennes factors and choosing appropriate lanthanide-contracted $c$ and $a$ values.
\end{abstract}

DOI: 10.1103/PhysRevLett.118.197202

Close scrutiny and an $a b$ initio description of the magnetism of rare earth materials are motivated by its increasing importance for many applications as well as the fundamental interest of the strongly correlated $f$ electrons underpinning it. A bench test for this task and an outstanding challenge in its own right is to explain the diverse magnetism of the heavy rare earth (HRE) elements.

The lanthanides from gadolinium to lutetium order into an apparently complex array of magnetic phases [1] despite the common chemistry of their valence electronic structure $\left(5 d^{1} 6 s^{2}\right.$ atomic configuration). Under ambient conditions, they crystallize into hexagonal close-packed structures, and the number of localized $f$ electrons per atom increases from seven for Gd's half-filled shell through to Lu's complete set of 14, which causes the lanthanide contraction of the lattice [2]. The magnetism is complicated. When cooled through $T_{c}$, Gd's paramagnetic (PM) phase undergoes a secondorder transition to a ferromagnetic (FM) state, whereas, at $T_{N}$, Tb, Dy, and Ho form incommensurate, helical antiferromagnetic (HAFM) phases where the magnetization spirals around the crystal $c$ axis. When the temperature is lowered further, both $\mathrm{Tb}$ and Dy undergo a first-order transition at $T_{t}$ to a FM phase with a basal plane orientation, and Ho forms a conical HAFM ground state.

Further exotic phases emerge when the metals are subjected to magnetic fields, and they have been extensively studied in experiments [3-10]. Below its $T_{c}$, Gd

Published by the American Physical Society under the terms of the Creative Commons Attribution 4.0 International license. Further distribution of this work must maintain attribution to the author(s) and the published article's title, journal citation, and DOI. preserves its FM order as the strength of the magnetic field applied along its easy axis is increased. This is in sharp contrast to Tb [3,11-13], Dy [4-7,14], and Ho [8,10] above $T_{t}$, which first distort their HAFM order (dis-HAFM) before undergoing a first-order transition into a fan magnetic structure followed by a second-order transition to a FM state with a further increase in the magnetic field. Dy and Ho also exhibit signs of additional spin-flip and vortex transitions associated with subtle changes in measured magnetization curves. In this Letter, we argue that much of this diversity stems directly from the valence electronic structure that all the HRE elements share.

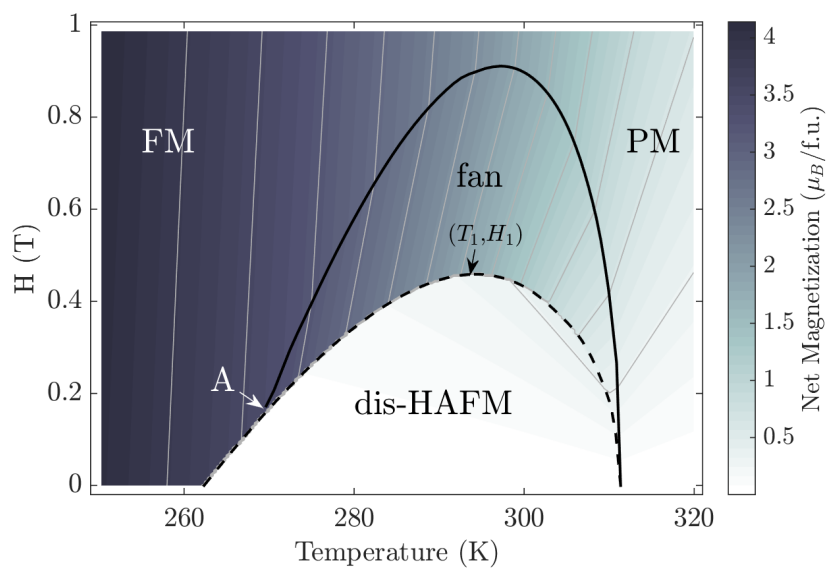

FIG. 1. The generic magnetic $T-H$ phase diagram for a heavy lanthanide metal for $\mathbf{H}$ applied along the easy direction constructed from the theory. Continuous (discontinuous) lines correspond to second- (first-) order phase transitions, and a tricritical point is marked $(A)$. The calculations were performed for the prototype $\mathrm{Gd}$ with lattice constants appropriate to Dy. 
Extensive experimental and theoretical investigations satisfactorily explain the onset of magnetic order from the PM state [15-21] via a detailed version of the famous Ruderman-Kittel-Kasuya-Yoshida (RKKY) pairwise interaction. The existence of nesting vectors $\mathbf{q}_{\text {nest }}$ separating parallel Fermi surface (FS) sheets of the valence electrons provokes a singularity in the conduction-electron susceptibility. This feature results in a $\mathbf{q}_{\text {nest }}$-modulated magnetic phase $[1,22]$, identified as a HAFM structure, incommensurate with the underlying lattice. The lanthanide contraction changes the FS topology $[16,20,23]$ and acts as the decisive factor for the emergence of the nesting vectors. This has resulted in the construction of a universal crystallomagnetic phase diagram which links the magnetic ordering that emerges from the PM state to the specific $c$ and $a$ lattice parameters of a heavy lanthanide system $[16,18]$.

The prominence of RKKY interactions in the discussion of lanthanide magnetism promotes a deeper inspection of the common HRE valence electronic structure. As magnetic order among the local $f$-electron moments of the HREs develops with a decreasing temperature and/or strengthening applied magnetic field, the valence-electron glue spinpolarizes and qualitatively changes. An indication of this was found by Khmelevskyi et al., who calculated effective exchange interactions from the FM state to be different from those in the PM state [24]. This effect has a potentially profound feedback on the interactions between the magnetic moments and a wider relevance for other magnetic systems where the physics is also typically couched in RKKY terms, including giant magnetoresistive nanostructures [25], rare earth clusters [26], magnetic semiconductors [27], and spin glasses [28]. We show how the response and feedback from the heavy lanthanide valence electrons to the ordering of local magnetic moments determine the main features of the magnetic phase diagrams. We establish a reference, summarized in Fig. 1, against which these magnetic properties can be analyzed to discriminate specific, subtle $f$-electron features.

A simple classical spin model with pairwise exchange interactions, magnetic anisotropy contribution, and a Zeeman external magnetic field term describes magneticfield-driven phase transitions in some antiferromagnetic insulators $[29,30]$. If the local moments of a HAFM state are pinned by anisotropy and crystal field effects to spiral around a particular direction, the effect of a magnetic field causes a first-order transition to a fan or conical phase where the spins now oscillate about the field direction. In higher fields, the fan or cone angles smoothly decrease to zero in the FM state. Such a model applied to the HREs addresses only part of the phenomenon, however, since it fails to reproduce the first-order dis-HAFM to FM and second-order fan to FM transitions at low temperatures and fields. It misses a tricritical point in consequence. In the seminal work by Jensen and Mackintosh [31] where the formation of field-induced fan and helifan phases was investigated theoretically for the first time, the key aspects of the HRE magnetic phase diagrams were reproduced only if ad hoc temperature-dependent pairwise exchange interactions were incorporated from a fit to spin wave measurements conducted at a series of temperatures. We find instead that much of the magnetic phase complexity is directly traced back to the behavior of the valence electrons. Evenson and Liu [15] maintained that the first-order HAFM-FM transition is driven by a magnetoelastic effect. We show rather that, while there is a magnetostructural coupling, it is not necessary for the transition.

We have extended the $a b$ initio density functional theory (DFT) -based, disordered local moment (DLM) approach [32] to address this issue and construct a generic $H-T$ magnetic phase diagram of the HREs. The DLM-DFT describes the effects of thermally induced "local moment" fluctuations on the underlying valence electronic structure of a magnet which itself generates the interactions between these moments. For many materials, the magnetic excitations can be modeled by allowing the orientations of local, in the case of the HRE's $f$ electron, moments to vary very slowly on the time scale of the valence electronic motions. By taking appropriate ensemble averages over their orientational configurations, DLM-DFT determines the system's magnetic properties and describes magnetic phase diagrams ab initio [33-35], temperature-dependent magnetic anisotropy [36-38], and field- and temperature-induced metamagnetic transitions [39]. Moreover, the valenceelectron-mediated, local moment interactions in the HREs depend directly on the extent of the magnetic order. This circumstance leads inevitably to "multispin" interactions which are not imposed from the outset but derive naturally from the $a b$ initio electronic basis of the theory [32] and are therefore tied to FS topology.

Gd is a convenient prototype system owing to its seven localized $f$ electrons per atom in an $S$ state which form a large moment and the small crystal field and spin-orbit coupling effects that are prevalent. The $L=0 f$-electron configuration means that the main valence-electron physics is unclouded by the actual details of the method used to describe the strong $f$-electron correlations. Choosing $\mathrm{Gd}$ deliberately, therefore, enables a clean extraction of the common HRE valence-electron effects on the magnetic properties. Selecting the $c$ and $a$ lattice constants for other elements makes the analysis appropriate to Tb, Dy, Ho, etc. Figure 1 shows the results for Gd using the lattice parameters appropriate to Dy [18].

As emphasized above, the theory has the advantage that valence electronic structure can be monitored as a function of local moment disorder. This is highly pertinent owing to the recent development of advanced time-dependent spectroscopy techniques. Time-resolved resonant $\mathrm{x}$-ray and ultrafast magneto-optical Kerr studies confirm the central tenet that the dynamics of the HRE corelike $f$ electrons are on a much longer time scale than the excitations of the valence electrons [40]. Time- and angle-resolved photoemission studies have 


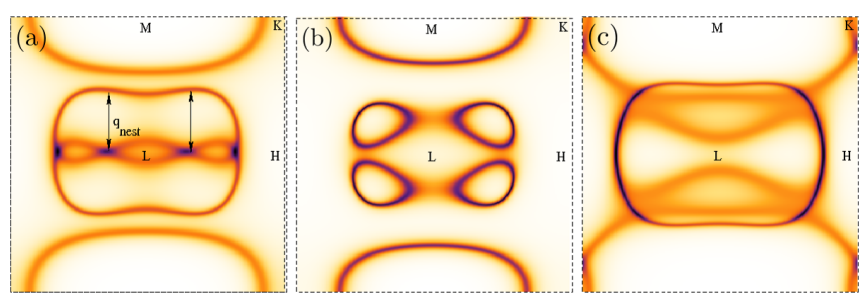

FIG. 2. The Bloch spectral function in the $L M H K$ plane at the Fermi energy for Gd with Dy's lattice constants for (a) the PM state and resolved into (b) majority spin and (c) minority spin components when there is an overall net average magnetization of $54 \%\left(m_{\mathrm{FM}}=0.54\right)$ of the $T=0 \mathrm{~K}$ saturation value in the FM state. This is the value in our calculations (Fig. 1) in the FM phase just below the temperature $T_{t}$ of the HAFM-FM first-order transition. $\mathbf{q}_{\text {nest }}$ indicates the nesting wave vector of the FS of the PM state, and the shading represents the broadening from thermally induced local moment disorder.

demonstrated the differing dynamics of spin-polarized valence states in correlated materials [41-43].

Figure 2 shows our calculated FS of Gd with Dy's lattice attributes within the DLM picture. Figure 2(a) shows the FS when the moments are randomly oriented in the paramagnetic state. The nesting vectors responsible for the onset of Dy's HAFM state below $T_{N}$ are clearly seen [18]. Figures 2(b) and 2(c) show the FS where now the local $f$-electron moments are oriented on average to produce an overall net average magnetization of $54 \%$ of the $T=0 \mathrm{~K}$ saturation value. The FS is spin-polarized, and neither the majority nor minority spin component continues to show nesting. This dramatic change of FS topology hints at the valence electron's role in the HAFM-FM metamagnetic transition. It also concurs with conclusions drawn from Döbrich et al.'s [19] angle-resolved photoemission measurements pointing to the magnetic exchange splitting of the FS as the principal mechanism for the fading of the nesting vectors [22] resulting in the stability of the FM phase at the ground state in $\mathrm{Tb}$ and $\mathrm{Dy}$.

To follow the repercussions of this insight, we specify a generalized grand potential $\Omega\left(\left\{\hat{e}_{n, i}\right\}\right)$ from the DFT in which the local moments are constrained to point along directions $\left\{\hat{e}_{n, i}\right\}$ [32]. This quantity is averaged over many such configurations with a probability distribution $P\left\{\hat{e}_{n, i}\right\}=\prod_{n} \prod_{i} P_{n}\left(\hat{e}_{n, i}\right)$, where $P_{n}\left(\hat{e}_{n, i}\right)=\left(\exp \left(\mathbf{A}_{n} \cdot \hat{e}_{n, i}\right) /\right.$ $\left.\int \exp \left(\mathbf{A}_{n} \cdot \hat{e}_{n, i}^{\prime}\right) d \hat{e}_{n, i}^{\prime}\right) . n$ and $i$ count over layers stacked along the $c$ axis (i.e., the $z$ axis) and sites within a layer, respectively. The local average $\mathbf{m}_{n}=\left\langle\hat{e}_{n, i}\right\rangle=\left[-\left(1 / A_{n}\right)+\right.$ $\left.\operatorname{coth} A_{n}\right] \hat{A}$ therefore defines an order parameter prescribed by the input $\left\{\mathbf{A}_{n}\right\}$ values. A magnetically ordered state is specified by the set $\left\{\mathbf{m}_{n}\right\}$. The PM state corresponds to $\left\{\mathbf{m}_{n}\right\}=\{\mathbf{0}\}$ and the FM state to $\left\{\mathbf{m}_{n}\right\}=\left\{\mathbf{m}_{\mathrm{FM}}\right\}$. An HAFM phase modulated by the wave vector $\mathbf{q}_{0}=\left(0,0, q_{0}\right)$ applies when $\mathbf{m}_{n}=m_{\mathrm{HAFM}}\left[\cos \left(\mathbf{q}_{0} \cdot \mathbf{R}_{n}\right), \sin \left(\mathbf{q}_{0} \cdot \mathbf{R}_{n}\right), 0\right]$, where $\mathbf{R}_{n}$ indicates the position of the $n$th layer. The average $\left\langle\Omega\left(\left\{\hat{\boldsymbol{e}}_{n, i}\right\}\right)\right\rangle$ is consequently a function of the $\left\{\mathbf{m}_{n}\right\}$ magnetic order parameters $\bar{\Omega}\left(\left\{\mathbf{m}_{n}\right\}\right)$. By repeating the calculation for many sets of $\left\{\mathbf{m}_{n}\right\}$ (i.e., $\left\{\mathbf{A}_{n}\right\}$ choices) and careful analysis [44], we discover that the internal magnetic energy $\bar{\Omega}$ has the following structure:

$$
\bar{\Omega}=-\sum_{n, n^{\prime}}\left(\mathcal{J}_{n n^{\prime}}+\sum_{n^{\prime \prime}, n^{\prime \prime \prime}} \mathcal{K}_{n n^{\prime}, n^{\prime \prime} n^{\prime \prime \prime}} \mathbf{m}_{n^{\prime \prime}} \cdot \mathbf{m}_{n^{\prime \prime \prime}}\right) \mathbf{m}_{n} \cdot \mathbf{m}_{n^{\prime}} .
$$

$\mathcal{J}_{n n^{\prime}}$ 's are interpreted as pairwise local moment interactions, whereas the quartic coefficients $\mathcal{K}_{n n^{\prime}, n^{\prime \prime} n^{\prime \prime \prime}}$ arise from the mutual feedback between local moment magnetic order and the spin-polarized valence electrons illustrated in Fig. 2. We found higher-order effective spin interaction terms to be vanishingly small.

For a phase diagram such as Fig. 1, we construct the system's Gibbs free energy $\mathcal{G}$ from

$$
\mathcal{G}=\bar{\Omega}-\sum_{n}\left[\mu \mathbf{m}_{n} \cdot \mathbf{H}+T S_{n}\left(\mathbf{m}_{n}\right)+F_{u}\left(\mathbf{m}_{n}\right)\right],
$$

where $T$ and $S_{n}\left(\mathbf{m}_{n}\right)=-k_{B} \int P_{n}(\hat{e}) \ln P_{n}(\hat{e}) d \hat{e}$ are the temperature and the magnetic entropy of the $n$th layer, respectively ( $k_{B}$ being Boltzmann's constant). The second term couples the external magnetic field $\mathbf{H}$ to the local magnetic moments each with magnitude $\mu$. The last uniaxial anisotropy term $F_{u}\left(\mathbf{m}_{n}\right)=F_{0}\left\langle\left(\hat{e}_{n, i} \cdot \hat{z}\right)^{2}\right\rangle$ [46], with strength $F_{0}$ [47], is there solely to fix the easy plane of magnetization. The phase diagram features are largely insensitive to its magnitude [44]. For selected $T$ and $\mathbf{H}$ values, the Gibbs free energy $\mathcal{G}$ is evaluated for the values $\left\{\mathbf{m}_{n}\right\}$ which minimize it, i.e., $\nabla_{\mathbf{m}_{i}} \mathcal{G}\left(\mathbf{m}_{i}, \mathbf{h}_{i} ; T\right)=\mathbf{0}$. This is accomplished when

$$
\mathbf{A}_{n}=-\beta\left[\nabla_{\mathbf{m}_{n}} \bar{\Omega}-\nabla_{\mathbf{m}_{n}} F_{u}\left(\mathbf{m}_{n}\right)-\mathbf{H}\right]=\beta \mathbf{h}_{n},
$$

where $\beta=1 / k_{B} T$. The $\mathbf{h}_{n}$ 's are therefore the Weiss fields for this mean field theory $[32,34,35,48]$.

We carried out DLM-DFT calculations [44] for the prototype $\mathrm{Gd}$ within this framework for the $c$ and $a$ lattice parameters appropriate to Gd, Tb, Dy, and Ho and in each case calculated the charge and magnetization densities self-consistently for the PM state $\left(\left\{\mathbf{m}_{n}=0\right\}\right)$. The selfinteraction correction was used to capture the strong correlations of the $f$ electrons $[18,49,50]$. A local moment of $\mu \approx 7.3 \mu_{B}$ established on each $\mathrm{Gd}$ site. We then divided the hexagonal lattice into 10 layer stacks and specified identical sets of $\left\{\mathbf{m}_{n}\right\}(n=1, \ldots, 10)$ values for each stack to define the magnetic order parameter for each layer. For each $c$ and $a$ pair, using the effective one-electron PM potentials, we calculated the $\mathbf{h}_{n}^{\prime}=-\nabla_{\mathbf{m}_{n}} \bar{\Omega}$ values for each $\left\{\mathbf{m}_{n}\right\}$ set. By thoroughly sampling the extensive $\mathbf{m}_{n}$ space, we tested and established a method [44] to extract the $\mathcal{J}_{n n^{\prime}}$ and $\mathcal{K}_{n n^{\prime}, n^{\prime \prime} n^{\prime \prime \prime}}$ constants of Eq. (1). We also checked that higher-order terms were vanishingly small [44]. Figure 1 summarizes the results for $\mathrm{Gd}$ with the lattice spacings appropriate to Dy. At a value $m_{\mathrm{FM}}=0.503$, the system undergoes a first-order transition from a HAFM to FM state 


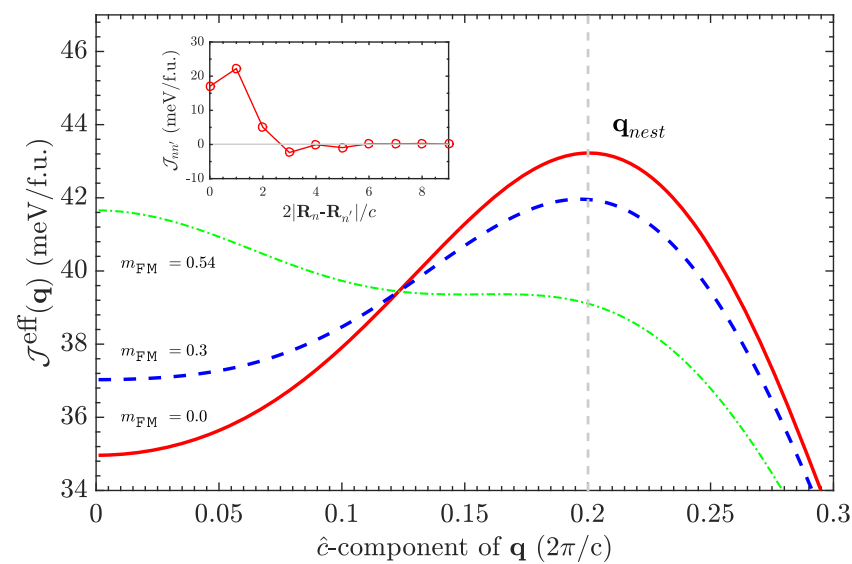

FIG. 3. The lattice Fourier transform of the effective pair interactions $\mathcal{J}^{\text {eff }}(\mathbf{q})$ (red line) when $m_{\mathrm{FM}}=0$ and its change when the FS is spin polarized, for finite $m_{\mathrm{FM}}$ (dashed blue line for $m_{\mathrm{FM}}=0.3$ and dot-dashed green line for $\left.m_{\mathrm{FM}}=0.54\right)$. The inset shows the dependence of the pair interactions $\mathcal{J}_{n n^{\prime}}$ on separation $R_{n n^{\prime}}=\left|\mathbf{R}_{n}-\mathbf{R}_{n^{\prime}}\right|$ for $m_{\mathrm{FM}}=0$.

in a zero field at $T_{t}=262 \mathrm{~K}$, which correlates with the FS topological changes depicted in Fig. 2. When the four-site $\mathcal{K}_{n n^{\prime}, n^{\prime \prime} n^{\prime \prime \prime}}$ 's are neglected in Eq. (1), the calculated phase diagram is very different [44], and a FM phase does not appear at low temperatures and fields.

In the presence of long-ranged magnetic order, quantified by $m_{\mathrm{FM}}$, effective pair interactions mediated by the valence electrons affected by the long-range magnetic order can be specified as $\mathcal{J}_{n n^{\prime}}^{\text {eff }}=\mathcal{J}_{n n^{\prime}}+\sum_{n^{\prime \prime} n^{\prime \prime \prime}} \mathcal{K}_{n n^{\prime}, n^{\prime \prime} n^{\prime \prime \prime}} m_{\mathrm{FM}}^{2}$ [44], and they incorporate the influence of the four-site terms from Eq. (1). Figure 3 shows their lattice Fourier transform relevant to Figs. 1 and 2 revealing the effect of the valence-electron spin polarization. As shown in the inset, for $m_{\mathrm{FM}}=0$, the interactions have a long-ranged oscillatory nature, so that $\mathcal{J}^{\text {eff }}(\mathbf{q})$ peaks at $\mathbf{q}_{\text {nest }} \approx 0.2(2 \pi / c) \hat{c}$ (full red line). This is a direct consequence of the FS nesting shown in Fig. 2(a) and which drives the HAFM magnetic order. We also show $\mathcal{J}^{\text {eff }}(\mathbf{q})$ for nonzero $m_{\mathrm{FM}}$. When $m_{\mathrm{FM}}=0.54$ (green, dot-dashed lines), the value in the FM phase just below $T_{t}, \mathcal{J}^{\text {eff }}(\mathbf{q})$ peaks at $\mathbf{q}=\mathbf{0}$, showing how the development of long-range magnetic order has favored the shift towards ferromagnetism. This confirms the role of the spin-polarized valence electrons and altered FS topology exemplified in Figs. 2(b) and 2(c).
By comparing Gibbs free energies of the FM, HAFM, conical, fan, and helifan structures obtained, we constructed the $T-H$ phase diagram. Figure 1 shows the results for Gd using $c$ and $a$ values appropriate to Dy when $\mathbf{H}$ was applied along the easy direction. Continuous (dashed) lines correspond to second- (first-) order phase transitions. We imposed a single-site uniaxial anisotropy of typical magnitude $F_{0}=+6 \mathrm{meV} /$ site [47] which precluded the conical phase when the magnetic field was applied in the easy $a b$ plane [44].

The figure reproduces all the main features that the experimentally measured magnetic phase diagrams of heavy lanthanide metals and their alloys have in common. There is the first-order HAFM-FM transition in the absence of $\mathbf{H}$ at $T_{t}$. Then for increasing values of $\mathbf{H}$ applied along the easy direction, the helical structure initially distorts before transforming to the fan structure. Increasing $\mathbf{H}$ further stabilizes the FM phase. There is, also in line with the experiment, a second-order transition from the fan to the FM phase in a finite field on cooling, and we find a tricritical point which is marked " $A$ " in Fig. 1.

We can adapt this Gd-prototype model to a specific heavy lanthanide element or alloy by using suitable lattice constant values for lanthanide-contracted Tb, Dy, and Ho [18] and accounting for the specific $f$-electron configuration. The $\mathrm{Gd}$ ion has orbital angular momentum $L=0$ and negligible spin-orbit coupling effects. $L S$ coupling, however, is important for the HREs. A simple measure to account for the different total angular momentum values, $J$, is to scale the $\mathcal{J}_{n n^{\prime}}$ and $\mathcal{K}_{n n^{\prime}, n^{\prime \prime} n^{\prime \prime \prime}}$ interactions with the famous de Gennes factor [1] $\left(g_{J}-1\right) 2 J(J+1)$, where $g_{J}$ is the Landé $g$ factor. We applied this treatment to Tb, Dy, and Ho, and each metal had a phase diagram of the form of Fig. 1 consistent with the experiment. For lattice parameters appropriate to $\mathrm{Gd}$, as characterized experimentally, the FS was topologically distinct from the other HREs with no nesting [18]. The local moments interactions were qualitatively different, leading to Gd's simple phase diagram as found experimentally.

In Table I, we compare results for $\mathrm{Tb}$, Dy, and $\mathrm{Ho}$ with those available from the experiment for $T_{N}$ and $T_{t}$ and the values of $T$ for the highest $\mathbf{H}$ for the dis-HAFM phase. We also theoretically estimate the tricritical point. The theory correctly captures trends and transition temperature

TABLE I. Application of the theory to Tb, Dy, and Ho and comparison with the experiment. The values of $T_{N}, T_{t}$, and the $T$ for the highest $\mathbf{H}$ for the dis-HAFM phase are compared to the experiment $\left(T_{1}\right.$ and $H_{1}$ in Fig. 1). A theoretical estimate of the tricritical point $(A)$ is also given. The theory also finds Gd to have a PM-to-FM second-order transition at $T_{C}=274 \mathrm{~K}$ ( $T_{C}=293 \mathrm{~K}$ in the experiment [1]). We remark that both the HRE metals $\mathrm{Er}$ and $\mathrm{Tm}$, which have larger lanthanide contractions, form incommensurate antiferromagnetic magnetic structures at low $T$ and show no transitions to FM states [1] in agreement with the trends predicted here.

\begin{tabular}{lccccccccccc}
\hline \hline Element & $T_{N}(\mathrm{~K})$ & $T_{N}^{\exp }(\mathrm{K})$ & $T_{t}(\mathrm{~K})$ & $T_{t}^{\exp }(\mathrm{K})$ & $T_{1}(\mathrm{~K})$ & $T_{1}^{\exp }(\mathrm{K})$ & $H_{1}(\mathrm{~T})$ & $H_{1}^{\exp }(\mathrm{T})$ & $T_{A}(\mathrm{~K})$ & $H_{A}(\mathrm{~T})$ & $\mathrm{References}$ \\
\hline $\mathrm{Tb}$ & 214 & 229 & 206 & 222 & 211 & $224-226$ & 0.03 & $0.02-0.03$ & 207 & 0.01 & {$[3,11-13]$} \\
$\mathrm{Dy}$ & 145 & 180 & 90 & 90 & 129 & $165-172$ & 0.43 & $1.1-1.2$ & 94 & 0.07 & {$[4,7,14]$} \\
Ho & 94 & 133 & $\cdots$ & 20 & 65 & 110 & 1.03 & 3.0 & $\cdots$ & $\cdots$ & {$[8,53,54]$} \\
\hline \hline
\end{tabular}


and field magnitudes. When the $c$ and $a$ values are further decreased, our model predicts that the FM phase does not appear at low $T$ and fields in accord with some experiments [51]. Discrepancies between the model and experiment can further highlight where $f$-electron correlation [52] effects are leading to more complicated physics. For example, the complex spin slip phases in Ho reported at low temperatures $[8,10]$ are not found in our model. The same applies to the vortex and helifan phases inferred from some experimental studies [7,31].

In summary, we claim a dominant role for the valence electrons in the temperature-field magnetic phase diagrams of the HRE metals. Our theory incorporates lattice structural effects coming from the lanthanide contraction on this glue and makes the link between the changing topology of the FS, observed experimentally, and the evolving longrange magnetic order of the $f$-electron moments which triggers the first-order transition between HAFM and FM states. Tricritical points are also predicted. From this generic valence-electron effect, magnetic-order-dependent interactions among the localized $f$-electron moments derive naturally. This rules out the necessity to invoke ad hoc temperature-dependent effective interactions or magnetostrictive effects. A simple de Gennes factor scaling of the interactions along with magnetocrystalline anisotropy to fix the easy magnetization plane enables this model to be applied broadly to HREs. We propose the model as a filter to identify subtle lanthanide $f$-electron correlation effects for further scrutiny.

The authors gratefully acknowledge discussions with O. Trushkevych and R. S. Edwards. The work was supported by Engineering and Physical Sciences Research Consul (United Kingdom) Grants No. EP/J006750/1 and No. EP/M028941/1.

*Corresponding author. e.mendive-tapia@warwick.ac.uk

[1] J. Jensen and A. R. Mackintosh, Rare Earth Magnetism, Estructure and Excitations (Clarendon, Oxford, 1991).

[2] K. A. Gschneidner and L. R. Eyring, Handbook on the Physics and Chemistry of Rare Earths (North-Holland, Amsterdam, 1978).

[3] V. I. Zverev, A. M. Tishin, A. S. Chernyshov, Y. Mudryk, K. A. Gschneidner, Jr., and V. K. Pecharsky, J. Phys. Condens. Matter 26, 066001 (2014).

[4] R. Herz and H. Kronmüller, J. Magn. Magn. Mater. 9, 273 (1978).

[5] M. T. Alkhafaji and N. Ali, J. Alloys Compd. 250, 659 (1997).

[6] Y. Kida, K. Tajima, Y. Shinoda, K. Hayashi, and H. Ohsumi, J. Phys. Soc. Jpn. 68, 650 (1999).

[7] A. S. Chernyshov, A. O. Tsokol, A. M. Tishin, K. A. Gschneidner, and V.K. Pecharsky, Phys. Rev. B 71, 184410 (2005).
[8] V. I. Zverev, A. M. Tishin, Z. Min, Y. Mudryk, K. A. Gschneidner, Jr., and V. K. Pecharsky, J. Phys. Condens. Matter 27, 146002 (2015).

[9] R. A. Cowley and S. Bates, J. Phys. C 21, 4113 (1988).

[10] N. Ali, F. Willis, M. O. Steinitz, M. Kahrizi, and D. A. Tindall, Phys. Rev. B 40, 11414 (1989).

[11] D. C. Jiles, S. B. Palmer, D. W. Jones, S. P. Farrant, and K. A. Gschneidner, J. Phys. F 14, 3061 (1984).

[12] D. C. Jiles, G. N. Blackie, and S. B. Palmer, J. Magn. Magn. Mater. 24, 75 (1981).

[13] G. I. Kataev, M. R. Sattarov, and A. M. Tishin, Phys. Status Solidi (a) 114, K79 (1989).

[14] J. Yu, P. R. LeClair, G. J. Mankey, J. L. Robertson, M. L. Crow, and W. Tian, Phys. Rev. B 91, 014404 (2015).

[15] W. E. Evenson and S. H. Liu, Phys. Rev. 178, 783 (1969).

[16] A. V. Andrianov, J. Magn. Magn. Mater. 140-144, 749 (1995).

[17] S. B. Palmer, G. J. McIntyre, A. V. Andrianov, and R. J. Melville, J. Magn. Magn. Mater. 177-181, 1023 (1998).

[18] I. D. Hughes, M. Däne, A. Ernst, W. Hergert, M. Lüders, J. Poulter, J. B. Staunton, A. Svane, Z. Szotek, and W. M. Temmerman, Nature (London) 446, 650 (2007).

[19] K. M. Döbrich, A. Bostwick, J. L. McChesney, K. Rossnagel, E. Rotenberg, and G. Kaindl, Phys. Rev. Lett. 104, 246401 (2010).

[20] A. V. Andrianov, O. A. Savel'eva, E. Bauer, and J. B. Staunton, Phys. Rev. B 84, 132401 (2011).

[21] L. Oroszlány, A. Deák, E. Simon, S. Khmelevskyi, and L. Szunyogh, Phys. Rev. Lett. 115, 096402 (2015).

[22] S. H. Liu, Handbook of the Physics and Chemistry of Rare Earths (North-Holland, Amsterdam, 1978), Vol. 1.

[23] S. B. Dugdale, J. Low Temp. Phys. 40, 328 (2014).

[24] S. Khmelevskyi, T. Khmelevska, A. V. Ruban, and P. Mohn, J. Phys. Condens. Matter 19, 326218 (2007).

[25] L. Zhou, J. Wiebe, S. Lounis, E. Vedmedenko, F. Meier, S. Blügel, P. H. Dederichs, and R. Wiesendanger, Nat. Phys. 6, 187 (2010).

[26] L. Peters, S. Ghosh, B. Sanyal, C. van Dijk, J. Bowlan, W. de Heer, A. Delin, I. D. Marco, O. Eriksson, M. I. Katsnelson, B. Johansson, and A. Kirilyuk, Sci. Rep. 6, 19676 (2016).

[27] H. Ohno, Science 281, 951 (1998).

[28] M. Ali, P. Adie, C. H. Marrows, D. Greig, B. J. Hickey, and R. L. Stamps, Nat. Mater. 6, 70 (2007).

[29] T. Nagamiya and K. Nagata, Prog. Theor. Phys. 27, 1253 (1962).

[30] T. Nagamiya and K. Nagata, Prog. Theor. Phys. 31, 1 (1964).

[31] J. Jensen and A. R. Mackintosh, Phys. Rev. Lett. 64, 2699 (1990).

[32] B. L. Gyorffy, A. J. Pindor, J. Staunton, G. M. Stocks, and H. Winter, J. Phys. F 15, 1337 (1985).

[33] J. B. Staunton and B. L. Gyorffy, Phys. Rev. Lett. 69, 371 (1992).

[34] J. B. Staunton, R. Banerjee, M. dos Santos Dias, A. Deak, and L. Szunyogh, Phys. Rev. B 89, 054427 (2014).

[35] L. Petit, D. Paudyal, Y. Mudryk, K. A. Gschneidner, Jr., V. K. Pecharsky, M. Lüders, Z. Szotek, R. Banerjee, and J. B. Staunton, Phys. Rev. Lett. 115, 207201 (2015). 
[36] J. B. Staunton, S. Ostanin, S. S. A. Razee, B. L. Gyorffy, L. Szunyogh, B. Ginatempo, and E. Bruno, Phys. Rev. Lett. 93, 257204 (2004).

[37] J. B. Staunton, L. Szunyogh, A. Buruzs, B. L. Gyorffy, S. Ostanin, and L. Udvardi, Phys. Rev. B 74, 144411 (2006).

[38] M. Matsumoto, R. Banerjee, and J. B. Staunton, Phys. Rev. B 90, 054421 (2014).

[39] J. B. Staunton, M. dos Santos Dias, J. Peace, Z. Gercsi, and K. G. Sandeman, Phys. Rev. B 87, 060404(R) (2013).

[40] M. C. Langner, S. Roy, A. F. Kemper, Y.-D. Chuang, S. K. Mishra, R. B. Versteeg, Y. Zhu, M. P. Hertlein, T. E. Glover, K. Dumesnil, and R.W. Schoenlein, Phys. Rev. B 92, 184423 (2015).

[41] M. Teichmann, B. Frietsch, K. Döbrich, R. Carley, and M. Weinelt, Phys. Rev. B 91, 014425 (2015).

[42] B. Andres, M. Christ, C. Gahl, M. Wietstruk, M. Weinelt, and J. Kirschner, Phys. Rev. Lett. 115, 207404 (2015).

[43] K. Krieger, J. K. Dewhurst, P. Elliott, S. Sharma, and E. K. U. Gross, J. Chem. Theory Comput. 11, 4870 (2015).

[44] See Supplemental Material at http://link.aps.org/ supplemental/10.1103/PhysRevLett.118.197202, which includes Ref. [45], for further information about the DLM theory, the construction of the magnetic phase diagram, details about the DLM-DFT data and the evaluation of the effective pair interactions, and the magnetic phase diagrams of $\mathrm{Gd}$ with the attributes of $\mathrm{Gd}, \mathrm{Tb}, \mathrm{Dy}$, and Ho.

[45] I. Hughes, Ph.D. thesis, University of Warwick, 2007.

[46] H. B. Callen and E. Callen, J. Phys. Chem. Solids 27, 1271 (1966).

[47] K. A. McEwen, Handbook on the Physics and Chemistry of Rare Earths (North-Holland, Amsterdam, 1978), Vol. 1, p. 411.

[48] J. Staunton, B. Gyorffy, A. Pindor, G. Stocks, and H. Winter, J. Magn. Magn. Mater. 45, 15 (1984).

[49] M. Lüders, A. Ernst, M. Däne, Z. Szotek, A. Svane, D. Ködderitzsch, W. Hergert, B. L. Györffy, and W. M. Temmerman, Phys. Rev. B 71, 205109 (2005).

[50] J. P. Perdew and A. Zunger, Phys. Rev. B 23, 5048 (1981).

[51] O. Trushkevych, Y. Fan, R. Perry, and R. Edwards, J. Phys. D 46, 105005 (2013).

[52] I. L. M. Locht, Y. O. Kvashnin, D. C. M. Rodrigues, M. Pereiro, A. Bergman, L. Bergqvist, A. I. Lichtenstein, M. I. Katsnelson, A. Delin, A. B. Klautau, B. Johansson, I. Di Marco, and O. Eriksson, Phys. Rev. B 94, 085137 (2016).

[53] W. C. Koehler, J. W. Cable, H. R. Child, M. K. Wilkinson, and E. O. Wollan, Phys. Rev. 158, 450 (1967).

[54] J. R. Gebhardt and N. Ali, J. Appl. Phys. 83, 6299 (1998). 